\title{
Analysis of a cAMP-responsive activator reveals a two-component mechanism for transcriptional induction via signal-dependent factors
}

\author{
Toshihiro Nakajima, ${ }^{1}$ Chiharu Uchida, ${ }^{1}$ Stephen F. Anderson, ${ }^{2}$ Jeffrey D. Parvin, ${ }^{2}$ and Marc \\ Montminy ${ }^{1,3}$ \\ ${ }^{1}$ Department of Cell Biology, Harvard Medical School, Boston, Massachusetts 02115 USA; ${ }^{2}$ Department of Pathology, \\ Harvard Medical School, Brigham and Women's Hospital, Boston, Massachusetts 02115 USA
}

We have examined the mechanism by which the cAMP-responsive factor CREB stimulates target gene expression following its phosphorylation at Ser-133. Using an in vitro transcription assay, we found that two signals were required for target gene activation: a phospho(Ser-133)-dependent interaction of CREB with RNA polymerase II via the coactivator CBP and a glutamine-rich domain interaction with TFIID via hTAF 130 . The adenovirus E1A oncoprotein was found to inhibit phospho(Ser-133) CREB activity by binding to CBP and specifically blocking recruitment of RNA Pol II to the promoter. Our results suggest that the recruitment of CBP-RNA Pol II complexes per se is not sufficient for transcriptional activation and that activator-mediated recruitment of TFIID is additionally required for induction of signal-dependent genes.

[Key Words: cAMP responsive activator; CREB; transcriptional activation; RNA Pol II; signal-dependent factors]

Received November 18, 1996; revised version accepted February 7, 1997.

cAMP stimulates the expression of numerous genes via the PK-A-mediated phosphorylation of cAMP response element binding (CREB) protein at Ser-133 (Gonzalez and Montminy 1989). Although phosphorylation has been shown to regulate a number of nuclear factors by enhancing their nuclear-targeting or DNA-binding activities, CREB belongs to a group of regulators whose transactivation potential is affected specifically (Gonzalez et al. 1991; Hagiwara et al. 1992).

The CREB transactivation domain is bipartite, consisting of constitutive and inducible activators, termed Q2 and the kinase-inducible domain (KID), respectively, which function cooperatively to induce target genes in response to cAMP stimulation (Brindle et al. 1993). The glutamine-rich Q2 domain has been shown to stimulate transcription via its association with a component of the TFIID fraction, the TBP-associated factor $\mathrm{dTAF}_{\mathrm{II}} 110$ (Ferreri et al. 1994). In contrast, the KID region appears to recruit the transcriptional apparatus via its phospho/Ser133)-dependent interaction with the related coactivators CREB-binding protein (CBP) and P300 (Chrivia et al. 1993; Parker et al. 1996).

Current evidence suggests that in addition to mediat-

\footnotetext{
${ }^{3}$ Corresponding author. Present address: Research Division, Joslin Diabetes Center, Boston, Massachussetts 02215 USA.

E-MAIL montminm@joslab.harvard.edu; FAX (617) 735-1928.
}

ing CREB activity, CBP and P300 also function as coactivators for other signal-dependent factors. In this regard, CBP and P300 have been shown to interact with a number of phosphorylation-dependent activators such as jun (Arias et al. 1994), STAT2 (Bhattacharya et al. 1996), and elk-1 (Janknecht and Nordheim 1996), as well as certain nuclear receptors, including RAR, RXR, and TR (Chakravarti et al. 1996; Kamei et al. 1996). In most cases, complex formation with CBP is signal dependent and occurs via a transactivation domain in each nuclear factor. The functional importance of these interactions has been illustrated by immunoneutralization studies in which microinjection of CBP antiserum could block agonist-dependent induction of appropriate reporter genes (Arias et al. 1994) and by transient transfection assays in which overexpression of CBP could potentiate activatordependent transcription (Kwok et al. 1994). The involvement of CBP and P300 in these signaling events has been further supported by studies in which overexpression of the adenovirus E1A oncoprotein was found to repress transcriptional induction of numerous genes following its association with a carboxy-terminal domain in both coactivators (Eckner et al. 1994; Arany et al. 1995; Lunblad et al. 1995).

In a recent study we observed that activation of the ras pathway blocks phospho(Ser-133) CREB-dependent transcription via the mitogen-dependent recruitment of pp $90_{\text {RsK }}$ to CBP (Nakajima et al. 1996). Remarkably, 
pp90 $0_{\text {RSK }}$ binds to the same region of CBP and P300 as the adenovirus E1A oncoprotein. And like E1A, binding of pp $90_{\mathrm{RSK}}$ to that region is sufficient to inhibit phospho(Ser-133) CREB-dependent transcription. E1A and pp $90_{\mathrm{RSK}}$ appear to inhibit transcription of cAMP-responsive genes without interfering with complex formation between phospho(Ser-133) CREB and CBP (Nakajima et al. 1996), suggesting that these repressors may interfere specifically with the ability of CBP to associate with proteins in the transcriptional apparatus.

In a recent series of biochemical experiments, we noted that CBP is a component of RNA polymerase II complexes (RPCs) in vivo (Kee et al. 1996). To evaluate the mechanism by which CBP mediates transcriptional induction of cAMP-responsive genes, we have reconstituted PK-A-dependent transcription via CREB in vitro using a partially purified RNA Pol II holoenzyme fraction that contains CBP and P300. We found that CBP mediated the recruitment of functional RNA Pol II activity to CREB in a phospho(Ser-133)-dependent manner. Remarkably, recruitment of CBP-RPCs did not suffice for transcriptional activation of cAMP-responsive genes: Association of CREB with TFIID via the TATA binding protein-associated factor $\mathrm{hTAF}_{\mathrm{II}} 130$ was also required. Our results point to a two-component mechanism for activation of cAMP-responsive genes via CREB.

\section{Results \\ $A C B P-R P C$ mediates induction of $c A M P$-responsive genes via phospho(Ser-133) CREB}

Previous work demonstrating that $\mathrm{CBP}$ associates with RNA Pol II (Kee et al. 1996) prompted us to examine whether partially purified complexes of RNA Pol II that contain CBP could support phospho(Ser-133) CREB-dependent transcription in vitro. Following fractionation of a Hela whole-cell extract over Bio-Rex 70 resin, we obtained an $0.6 \mathrm{M}$ potassium acetate eluate fraction that was enriched eightfold in CBP-associated RNA Pol II activity relative to total protein, as revealed by in vitro transcription and Western blot assay of CBP immunoprecipitates (Fig. 1A,B). Western blot analysis of CBP immunoprecipitates prepared from this $0.6 \mathrm{M}$ fraction indicated that CBP was also associated with the Pol II holoenzyme component SRB7 (Fig. 1B).

To determine whether the CBP-Pol II complex could support phospho(Ser-133)-dependent CREB activity on a cAMP-responsive template, we performed in vitro transcription assays using purified basal transcription factors (TFIIA, TFIIB, TFIIE, TFIIF, TFIIH) and a phosphocellulose Hela TFIID fraction, plus the $0.6 \mathrm{~m}$ Bio-Rex fraction. Under these conditions, phospho(Ser-133) CREB was found to induce transcription from a $3 \times$ CRE-adenovirus major late promoter (MLP) template $\sim 10$-fold, whereas unphosphorylated CREB protein had no effect on promoter activity (Fig. 1C, cf. lanes 4 and 6). A mutant CREB polypeptide containing a Ser/Ala-133 substitution at the PK-A phosphoacceptor site was unable to stimulate transcription of the $3 \times$ CRE reporter in the presence of PK-A (Fig. 1C, lane 12), revealing the importance of Ser-133 for transcriptional induction in vitro. Mutant CREB polypeptides lacking the glutamine-rich Q2 domain were similarly inactive, indicating that, in addition to Ser-133 phosphorylation, Q2 was also important for CREB activity (Ferreri et al. 1994).

To test whether phospho(Ser-133) CREB stimulates target gene expression via a CBP-dependent mechanism in vitro, we added a dominant-negative CBP polypeptide that contains the CREB-binding domain (KIX) and that has been shown to block induction of cAMP-responsive genes when microinjected into fibroblasts (Parker et al. 1996). In vitro transcription reactions containing KIX peptide were unable to stimulate $3 \times$ CRE reporter activity via phospho(Ser-133) CREB, indicating that CBP is indeed required for PK-A-mediated transcription in vitro (Fig. 1C, cf. lanes 6 and 7). In control assays performed on immunoprecipitates of RNA Pol II prepared with antiCTD antiserum, addition of KIX did not inhibit basal transcription from the MLP template, revealing that the KIX peptide does not block RNA Pol II or basal factor activities (Fig. 1D, right panel).

Adenovirus ElA oncoprotein has been shown to inhibit phospho(Ser-133) CREB-dependent activation by binding to a conserved carboxy-terminal domain in CBP and $\mathrm{P} 300$ via residues in conserved region 1 (CR1) and amino terminus of E1A (Eckner et al. 1994). Addition of an E1A polypeptide spanning exon 1 (amino acids 1-139) to in vitro transcription reactions also inhibited phospho(Ser-133) CREB-dependent activation of the $3 \times$ CRE template (Fig. 1C, lane 9). Phospho(Ser-133) CREB remained fully active, however, following addition of the mutant dl 1101 E1A polypeptide (Egan et al. 1988) containing a deletion in CR1 (amino acids 4-25) that abolishes interaction with CBP or P300 (Fig. 1C, lane 10). But neither wild-type nor mutant E1A polypeptides were found to interfere with RNA Pol II or basal factor activities in control assays performed with an MLP template on RNA Pol II immunoprecipitates (Fig. 1D, left panel).

\section{The CBP-RPC contains holoenzyme-associated proteins}

To further characterize the CBP-RPC, we fractionated the $0.6 \mathrm{M}$ Bio-Rex 70 column eluate over a $10 \%-60 \%$ sucrose gradient. In vitro transcription assay of CBP immunoprecipitates from individual gradient fractions revealed that the CBP-RPC was concentrated primarily in fractions $11-17$ and was purified $\sim 40$-fold relative to total protein (Fig. 2A, middle panel). CDK8, a homolog of yeast SRB10 and a component of the mammalian RNA Pol II holoenzyme (Maldonado et al. 1996), was found to sediment with the CBP-RPC in fractions 11-17, suggesting that the CBP-RPC may correspond to a form of RNA Pol II holoenzyme (Fig. 2A, top panel). TFIIE, TFIIF, and TFIIH activities could also be detected within the same peak fractions of CBP-Pol II activity by in vitro transcription assays, whereas TFIIB and TFIID activities could not (not shown). The distribution of TFIIH activity, for example, coincided with the peaks of CBP-RNA 
Pol II activity and CDK8 protein (Fig. 2A, bottom panel). In activation assays with a $3 \times$ CRE-MLP template, peak sucrose gradient fractions were also capable of mediating phospho(Ser-133) CREB-dependent activation in conjunction with a highly purified TFIID fraction (eTFIID) (Zhou et al. 1992) (Fig. 2B). Consistent with a coactivator requirement for recruitment of both RNA Pol II and TFIID by phospho(Ser-133) CREB, neither core RNA Pol II (Fig. 2B, cf. lanes 1 and 3) nor TBP were able to substitute for the holo-Pol II and TFIID fractions in transcription assays with the $3 \times$ CRE-MLP template (Fig. 2B, cf. lanes 2 and 3 ).

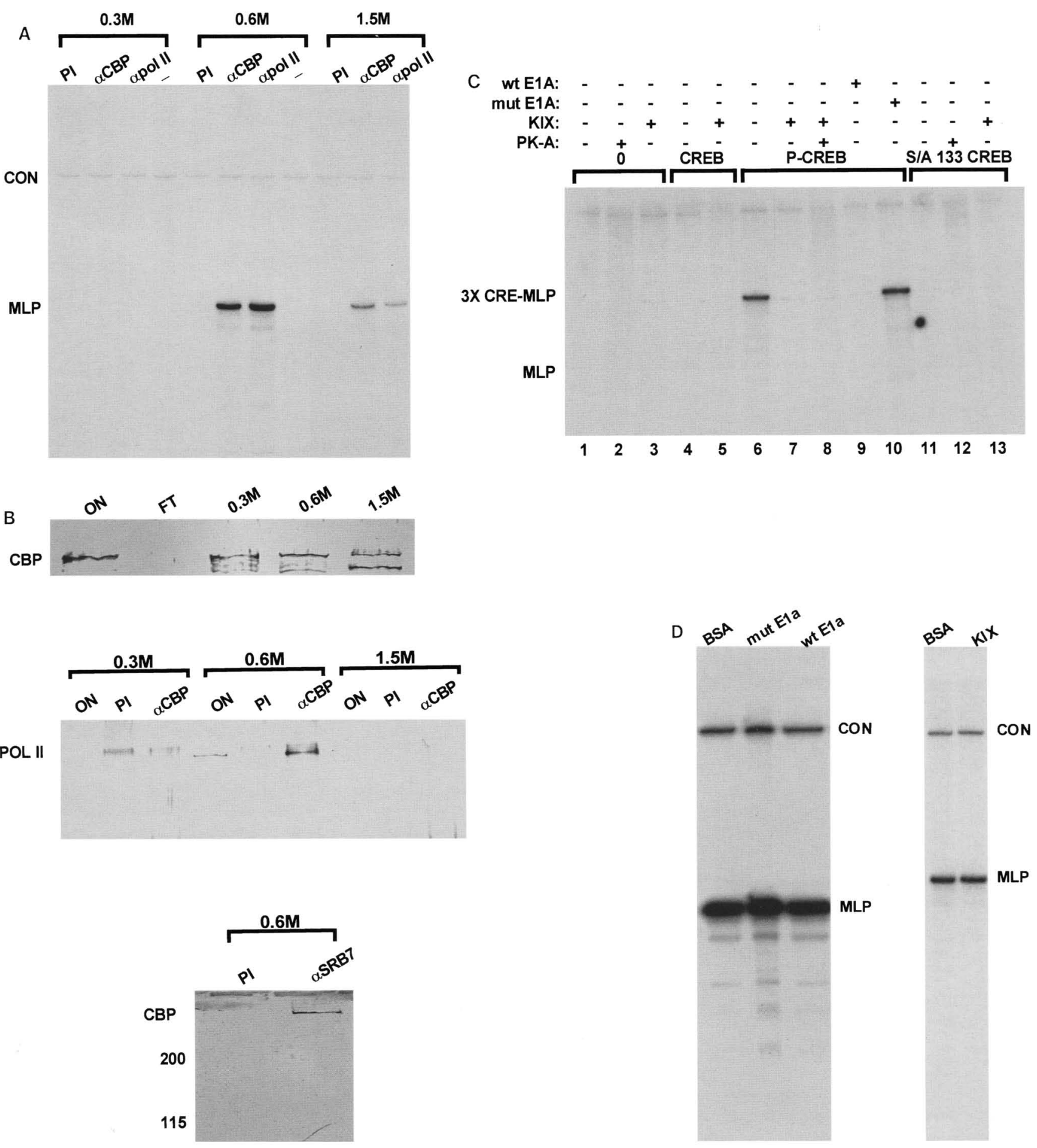

Figure 1. (See facing page for legend.) 
Phospho(Ser-133)CREB recruits RNA Pol II and TFIID activities

To test the prediction that phospho(Ser-133) CREB stimulates target gene expression by recruiting RNA Pol II activity via CBP, we performed affinity selection assays on the $0.6 \mathrm{M}$ Bio-Rex fraction with biotinylated CREB and phospho(Ser-133) CREB proteins. Following incubation with the $0.6 \mathrm{M}$ fraction, CREB protein complexes bound to avidin beads were washed, and binding of RNA Pol II activity to CREB and phospho(Ser-133) CREB was examined by an in vitro transcription assay using an MLP template plus all purified basal transcription factors except RNA Pol II. Phospho(Ser-133) CREB protein $(\mathrm{P})$ but not unphosphorylated CREB (U) was found to recruit RNA Pol II activity from the $0.6 \mathrm{M}$ BioRex column fraction (Fig. 3, lanes 5-8).

When incubated with samples containing a phosphocellulose HeLa TFIID fraction, unphosphorylated CREB and phospho(Ser-133) CREB beads were found to associate comparably with TFIID activity, as revealed by in vitro transcription assays containing purified basal factors minus TATA binding protein (TBP) (Fig. 3, lanes 9-12). No TBP recruitment was noted when affinity selection assays were performed using purified TBP in place of TFIID, however, demonstrating the importance of TBP-associated factors (TAFs) for this interaction (not shown).

Following affinity selection assay with the $0.6 \mathrm{~m}$ BioRex fraction supplemented with the Hela phosphocellulose TFIID fraction, phospho(Ser-133) CREB resin was found to recruit both TFIID and Pol II activities by in vitro transcription assay (Fig. 3, lane 4).
Phospho(Ser-133) CREB associates with RNA Pol II and TFIID via the KID and Q2 domains, respectively

Previous data showing that KID mediates association of phospho(Ser-133) CREB with CBP (Parker et al. 1996) prompted us to examine whether RNA Pol II activity is recruited to CREB via this domain. In affinity selection assays, glutathione $S$-transferase (GST)-phospho(Ser133) KID fusion protein was found to recruit RNA Pol II activity from the $0.6 \mathrm{M}$ Bio-Rex fraction but GST-Q2 did not (Fig. 4A, cf. lanes 2 and 3). In Western blot assays, GST-phospho(Ser-133) KID resin also recruited RNA Pol II large subunit, whereas unphosphorylated GST-KID resin did not (not shown). These results indicate that the KID domain mediates recruitment of RNA Pol II activity to phospho(Ser-133) CREB.

Although the KID region mediates transcriptional induction in response to PK-A, the inability of CREB Q2deletion mutants to stimulate CRE-dependent transcription (Brindle et al. 1993) suggests the requirement for a second signal in addition to recruitment of CBP. Previous data showing that $\mathrm{Q} 2$ specifically interacts with one component of the TFIID fraction $\left(\mathrm{dTAF}_{\mathrm{II}} 110\right)$ but not others (TBP, $\mathrm{dTAF}_{\mathrm{II}} 40, \mathrm{dTAF}_{\mathrm{II}} 80$ ) (Ferreri et al. 1994) prompted us to examine whether TFIID is functionally recruited to CREB via this coactivator. In affinity selection assays with GST fusion proteins containing either the Q2 or KID domains of CREB, GST-Q2 but not GSTphospho(Ser 133) KID resin was found to associate with TFIID by recruitment/in vitro transcription assay /Fig. $4 \mathrm{~B}$, cf. lanes 2-5). These results support the notion that KID and Q2 function as independent activators that synergize in response to cAMP stimulation (Brindle et al.

Figure 1. Reconstitution of phospho(Ser-133) CREB-dependent transcription in vitro. (A) In vitro transcription assays of Hela wholecell extract following fractionation over Bio-Rex 70 resin with increasing concentrations of potassium acetate buffer $[0.3 \mathrm{M}, 0.6 \mathrm{M}, 1.5$ $\mathrm{M})$ as indicated. Bio-Rex column fractions were evaluated for RNA Pol II activity following immunoprecipitation with preimmune (PI), anti-CBP $(\alpha \mathrm{CBP})$, or anti-RNA Pol II ( $\alpha$ pol II) antisera. RNA Pol II activity in each precipitate was evaluated by in vitro transcription assay with an adenovirus MLP template in reactions containing all purified basal transcription factors (TFIIB, TBP, TFIIE, TFIIF, TFIIH) minus RNA Pol II. (MLP) The 210-nucleotide transcript from G-less cassette placed downstream of MLP; (CON) 550-nucleotide ${ }^{32} \mathrm{P}$-labeled RNA added to each reaction following transcription assay as recovery control. $(B$, top $)$ Western blot analysis of CBP in 0.3 M, $0.6 \mathrm{M}, 1.5 \mathrm{M}$ potassium acetate eluate fractions following chromatography of HeLa whole-cell extract over Bio-Rex 70 resin. CBP antiserum 5614, a rabbit polyclonal antiserum against the CREB-binding domain of CBP, was used for Western blot assay. (Middle) Western blot assay of RNA Pol II associated with CBP in each Bio-Rex fraction following coimmunoprecipitations with 5614 antibody developed against the CREB-binding domain of CBP. Nonspecific bands in lanes 2 and 3 do not comigrate with RNA Pol II and represent edge of separator gel. Anti-CTD antiserum was used to detect RNA Pol II large subunit in this and other assays for RNA Pol II. (ON) Twenty percent of total onput protein used in immunoprecipitation assay; (PI) preimmune serum; $(\alpha \mathrm{CBP})$ anti-CBP antiserum. (Bottom) Western blot analysis of CBP following immunoprecipitation of $0.6 \mathrm{M}$ eluate fraction with anti-SRB7 or preimmune antiserum. $(C)$ In vitro transcription assays of CREB and phospho(Ser-133) CREB activity on 3× CRE-MLP template fused to a 390-bp G-less cassette. All reactions contained 0.6 M KAc Bio-Rex column fraction, which is enriched in CBP-RPCs and these reactions were supplemented with basal transcription factors (TFIIA, TFIIB, TFIID, TFIIE, TFIIF, TFIIH) minus RNA Pol II. Unphosphorylated CREB (CREB), phospho(Ser-133) CREB (P-CREB), or mutant Ser/Ala 133 CREB (S/A 133 CREB) were added as indicated. Addition of wild-type or mutant E1A polypeptides (wt E1A, mut E1A) as indicated; (KIX) 80-amino-acid CBP polypeptide extending from amino acids 591-670 and containing the CREB-binding domain of CBP; (PK-A) purified catalytic subunit of cAMP-dependent protein kinase A. Bands correspond to 390-nucleotide G-less transcript from 3× CRE-MLP template. Lane numbers indicated at bottom. The 210nucleotide transcript from a core MLP template (MLP) was not visible on this exposure. Prolonged exposure revealed that the basal transcript was constant. (D) KIX and E1A polypeptides do not interfere directly with RNA Pol II activity. In vitro transcription assays of RNA Pol II immunoprecipitates prepared from the $0.6 \mathrm{M}$ KAc fraction with anti-CTD antiserum. Addition of BSA, dl 1101 mutant E1A, wild-type E1A, or KIX polypeptides as indicated. Recovery control and 210-nucleotide MLP transcripts are indicated. No RNA Pol II activity was detected in control reactions using preimmune serum (Fig. 1B). 
A
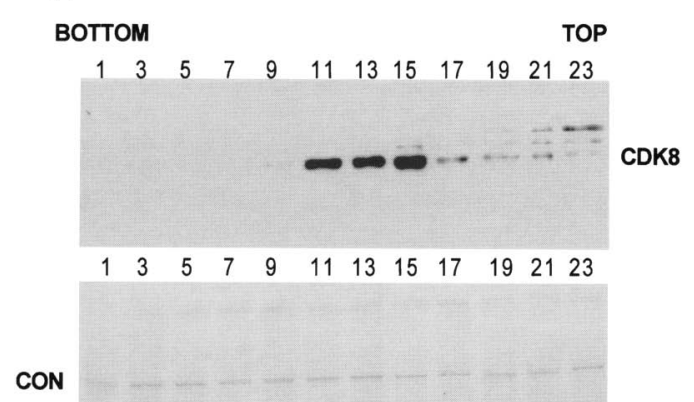

MLP

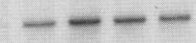

MLP

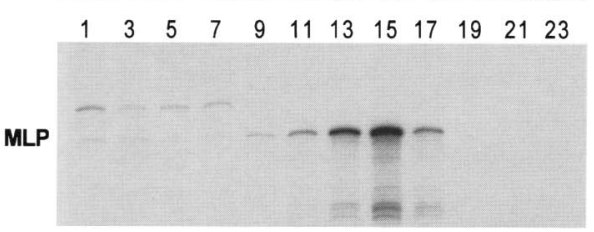

B
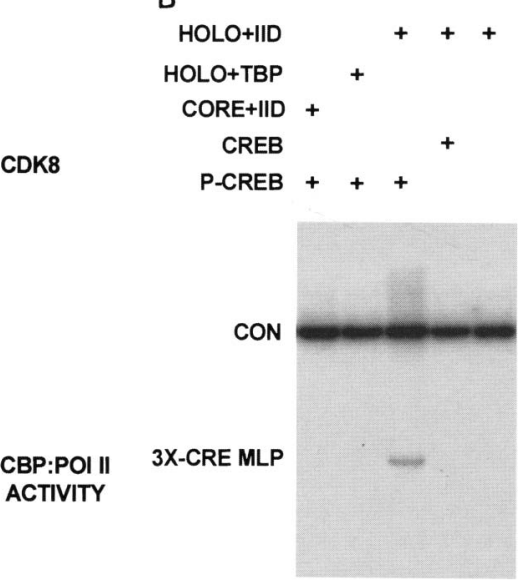

Figure 2. (A) The CBP-RPC cofractionates with certain general transcription factors and components of the mammalian Pol II holoenzyme complex. Purification of the $0.6 \mathrm{~m}$ Bio-Rex 70 column fraction over a $35-\mathrm{ml} 10 \%-60 \%$ gradient. One-milliliter sedimentation fractions were collected, and numbers above each lane correspond to odd-numbered fractions. (Top) Western blot assay of sucrose gradient fractions using antiserum against mammalian RNA Pol II holoenzyme component CDK8. (Middle) In vitro transcription assay of CBP immunoprecipitates prepared from odd-numbered sucrose gradient fractions. Immunoprecipitates were washed and then analyzed for RNA Pol II activity on an MLP template in reactions containing all purified general transcription factors (TFIIB, TBP, TFIIE, TFIIF, TFIIH) except RNA Pol II. Recovery control (CON) and MLP transcripts as shown. (Bottom) In vitro transcription assay of TFIIH activity in individual sucrose gradient fractions using linearized MLP template. In vitro transcription assays were performed with MLP template plus all purified GTFs minus TFIIH. $(B)$ CBP-RNA Pol II and TFIID complexes are both required for phospho(Ser-133) CREB-dependent activation of a cAMPresponsive template. In vitro transcription assay of $3 \times$ CRE-MLP template in reactions containing purified basal transcription factors (TFIIA, TFIIB, TFIIE, TFIIF, TFIIH) plus either highly purified Hela TFIID (eTFIID; IID) or recombinant TATA-binding protein (TBP). RNA Pol II activity was supplied either as a sucrose gradient purified holoenzyme fraction (HOLO) or core RNA polymerase (CORE). Addition of recombinant phospho(Ser-133) CREB or unphosphorylated CREB, as indicated. (CON) The 550-nucleotide ${ }^{32}$ P-labeled RNA used as recovery control.

1993) by virtue of their ability to recruit distinct factors in the transcriptional apparatus.

\section{The Q2 domain recruits TFIID activity via $h T A F_{I I} 130$}

The recent characterization of cDNA clones for hTAF $_{\text {II }} 130$ (Tanese et al. 1996), the human homolog of Drosophila dTAF $_{\text {II }} 110$, led us to evaluate whether the Q2 domain recruits TFIID via its association with this protein. In affinity selection assays with ${ }^{35} \mathrm{~S}$-labeled hTAF $_{\text {II }} 130$, Q2 but not phospho(Ser-133) KID was found to associate with $\mathrm{hTAF}_{\mathrm{II}} 130$ (Fig. $5 \mathrm{~A}$, cf. lanes 3 and 4). Moreover, both CREB and phospho(Ser-133) CREB associated comparably with $\mathrm{hTAF}_{\mathrm{II}} 130$, demonstrating the phosphorylation-independent nature of this interaction (Fig. 5A, lanes 5,6). To determine whether hTAF $_{11} 130$ was required for recruitment of TFIID activity via $Q 2$, we added affinity-purified $\mathrm{hTAF}_{\mathrm{II}} 130$ antiserum to pulldown reactions containing GST-Q2 resin plus crude TFIID fraction. Although preimmune antiserum had no effect on the ability of GST-Q2 to recruit TFIID activity by in vitro transcription assay, addition of hTAF 130 antiserum strongly inhibited association of TFIID activity with Q2 (Fig. 5B, cf. lanes 2 and 3). Addition of $\mathrm{hTAF}_{\mathrm{II}} 130$ antiserum to in vitro transcription reactions also blocked the ability of phospho(Ser-133) CREB to stimulate transcription from a $3 \times$ CRE MLP (Fig. 5C, cf. lanes 5 and 6). TAFF $_{\text {II }} 130$ antiserum did not inhibit basal

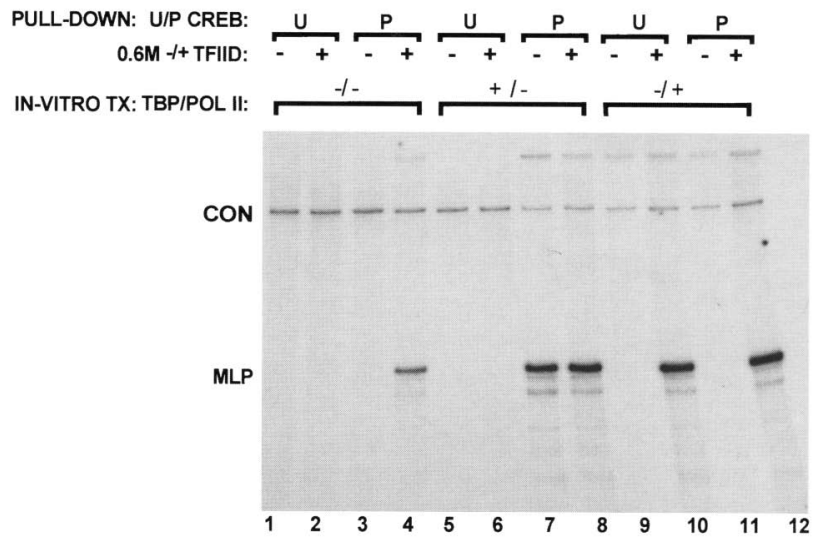

Figure 3. CREB stimulates transcription of cAMP-responsive genes by recruiting RNA Pol II and TFIID activities. In vitro transcription assay of biotinylated CREB (U) and phospho(Ser133) CREB (P) complexes following affinity selection with $0.6 \mathrm{M}$ Bio-Rex fraction alone (-TFIID) or supplemented with Hela phosphocellulose TFIID fraction (+TFIID). Following affinity selection with CREB and phospho(Ser-133) CREB avidin beads, recruitment of TFIID and RNA Pol II to CREB or phospho(Ser133) CREB resins was evaluated by in vitro transcription assay with purified basal factors minus both RNA Pol II and TBP $(-\mid-)$, minus RNA Pol II alone $(+\mid-)$, or minus TBP alone $(-1+)$. In this and in subsequent assays, avidin beads alone did not recruit either Pol II or TFIID activities detectably (not shown). The autoradiograph shows 210-nucleotide transcript from MLP template (MLP). Reactions were supplemented with ${ }^{32} \mathrm{P}$-labeled 550 -nucleotide RNA as recovery control (CON). 
A

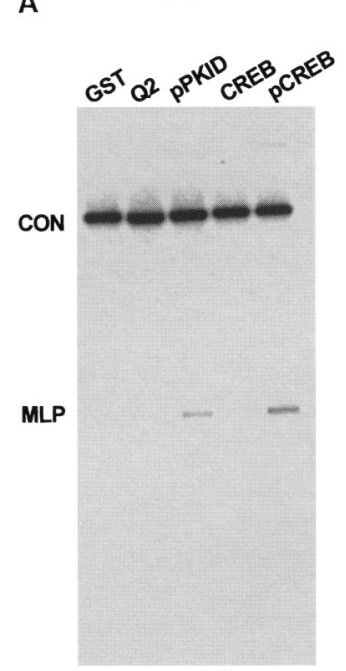

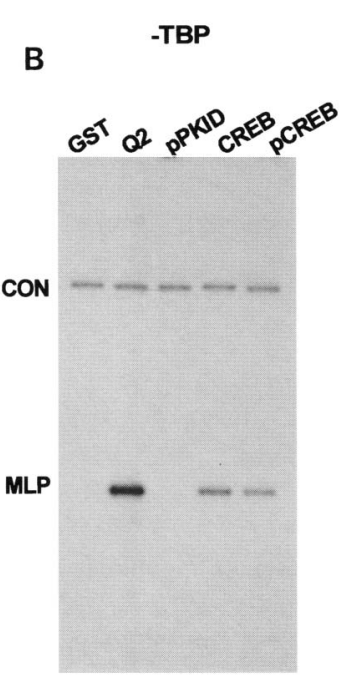

Figure 4. The KID and $\mathrm{Q} 2$ domains of CREB recruit RNA Pol II and TFIID activities, respectively. $(A)$ Recruitment of RNA Pol II to phospho(Ser-133) CREB occurs via KID but not the Q2 domain. In vitro transcription analysis of biotinylated CREB, phospho(Ser-133) CREB, or GST-fused phospho(Ser-133) KID (pPKID), and Q2 resins following pull-down assay with $0.6 \mathrm{M}$ Bio-Rex fraction. Assays were performed with MLP template and all purified basal transcription factors minus RNA Pol II (-POL II). (B) The glutamine-rich Q2 domain but not the phospho(Ser-133) kinase inducible domain (KID) in CREB associates with TFIID. Analysis of TFIID activity recovered from partially purified Hela TFIID fraction following affinity selection assay with biotinylated CREB or phospho(Ser-133) CREB (pCREB) or with GST fusion proteins containing GST alone, fused to Q2 (amino acids 160-283), or to Ser-133 phosphorylated KID (pKID) (amino acids 100-160). Recruitment of TFIID activity was evaluated by in vitro transcription assays in which all purified basal factors were provided except for TBP (-TBP). Transcript from MLP promoter template and recovery control (CON) as indicated.

transcription from an MLP template, however, demonstrating that this reagent does not block TFIID activity per se. These results reveal that the association of CREB with TFIID via $\mathrm{hTAF}_{\mathrm{II}} 130$ is required for transcriptional induction by protein kinase $\mathrm{A}$ (PK-A).

\section{E1A blocks phospho(Ser-133) CREB activity by disrupting $C B P-R P C s$}

To evaluate the mechanism by which E1A blocks activation of cAMP-responsive genes via phospho(Ser-133) CREB, we examined the effects of this oncoprotein on recruitment of RNA Pol II via the KID region. Recruitment/in vitro transcription assays with GST-phospho(Ser-133) KID resin revealed that wild-type but not $d 1$ 1101 mutant ElA polypeptide blocked association of phospho-KID with RNA Pol II activity (Fig. 6A, cf. lanes 3 and 4). E1A did not appear to interfere directly with RNA Pol II activity, however, as determined by analysis of immunoprecipitates of RNA Pol II recovered from the
0.6 M Bio-Rex fraction with anti-carboxy-terminal domain (CTD) antiserum (Fig. 1D, left panel).

To distinguish whether E1A disrupts recruitment of RNA Pol II activity to phospho(Ser-133) CREB by interfering with CBP-phospho(Ser133) KID or CBP-RNA Pol II interactions, we performed Western blot assays of GST-phospho(Ser-133) KID resins after pull-down assay

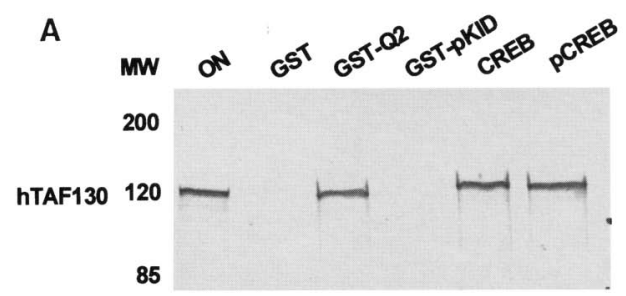

C

B

MLP

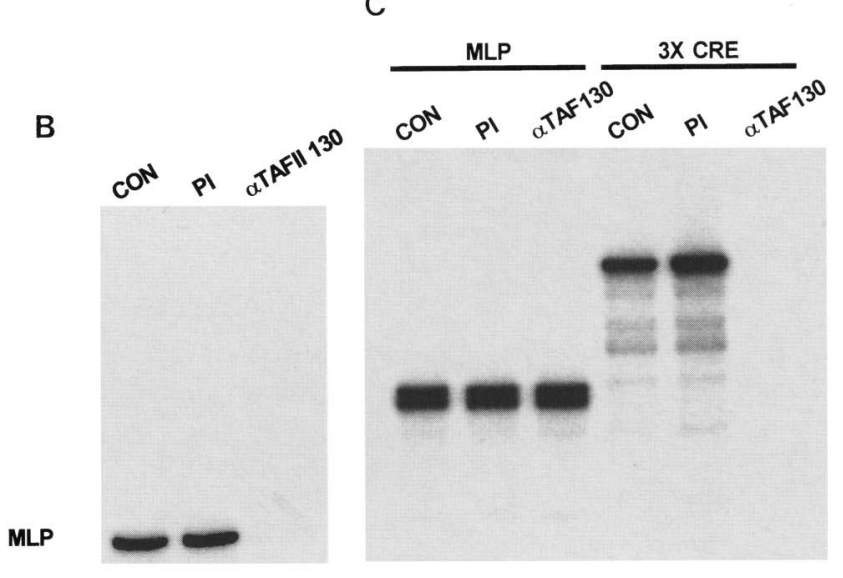

Figure 5. Recruitment of TFIID via Q2-hTAF 130 interaction is required for target gene activation by phospho(Ser-133) CREB. (A) Affinity selection assay of recombinant ${ }^{35} \mathrm{~S}$-labeled hTAF $_{\Pi} 130$. (ON) Twenty-five percent of total hTAF 130 onput; (GST) GST polypeptide; (GST-Q2) GST fusion protein containing Q2 domain (amino acids 160-283) of CREB; (GST-pKID) fusion protein containing phospho(Ser-133) KID (amino acids 100-160) domain of CREB; (CREB and pCREB) biotinylated recombinant CREB and phospho(Ser-133) CREB proteins. CREB and pCREB complexes were retrieved with avidin beads. (B) In vitro transcription assay of GST-Q2 resin following pull-down assay with phosphocellulose TFIID fraction. Prior to affinity selection, TFIID-containing samples were incubated on ice either with no antiserum (CON), preimmune serum (PI), or affinity-purified anti-hTAF 130 antiserum. Transcription reactions on glutathione beads were performed with MLP template plus all general transcription factors minus TBP. $(C) \mathrm{hTAF}_{\mathrm{II}} 130$ is required for phospho-CREB-dependent activation. In vitro transcription assay of MLP (lanes 1-3) and 3x CRE-MLP templates (lanes 4-6). Transcription reactions contained the $0.6 \mathrm{M}$ Bio-Rex column fraction, purified basal factors (TFIIA, TFIIB, TFIIE, TFIIF, TFIIH), and a phosphocellulose TFIID fraction. Reactions with $3 \times$ CRE-MLP template also contained $60 \mathrm{ng}$ of phospho(Ser-133) CREB. Prior to transcription reaction, TFIID samples were preincubated on ice with no antiserum, preimmune serum (PI), or affinity-purified anti-hTAF 130 antiserum as indicated over each lane. The 210 -nucleotide transcript from MLP template and 390-nucleotide transcript from 3× CRE-MLP template are shown. 


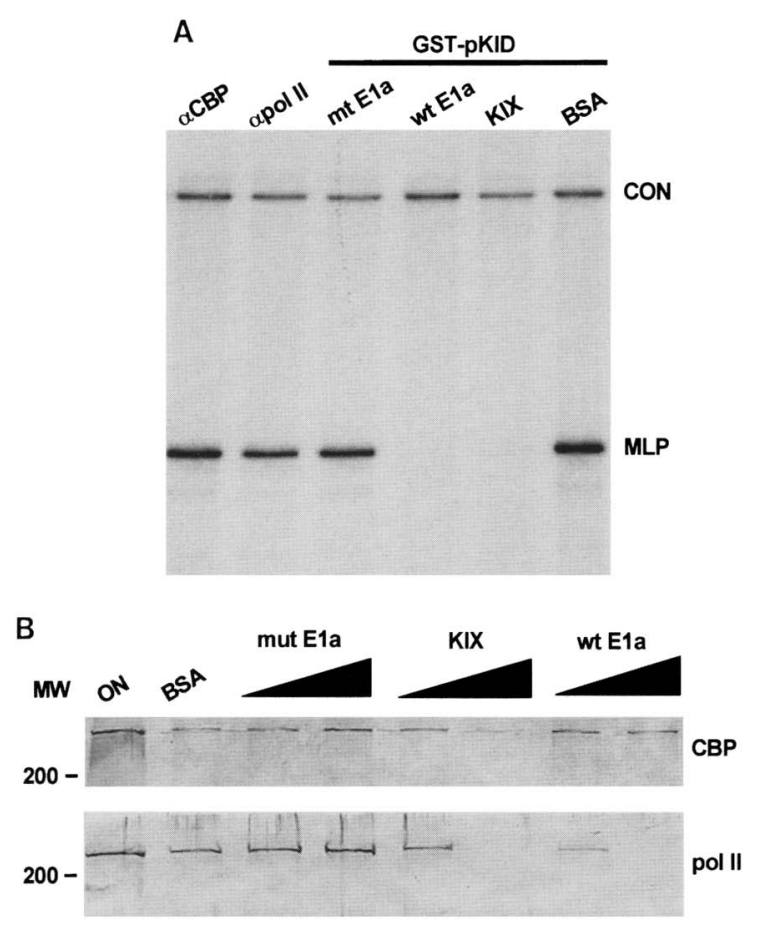

Figure 6. Adenovirus E1A oncoprotein blocks recruitment of RNA Pol II activity to phospho(Ser-133) KID domain of CREB. (A) In vitro transcription assay of GST-phospho(Ser-133) KID resins following affinity selection assay with $0.6 \mathrm{M}$ Bio-Rex fraction. Effect of wild-type Ela (wt E1A), mutant dl 1101 E1A (mt E1A) polypeptide that is unable to bind to $\mathrm{CBP}$, KIX polypeptide (which contains the CREB-binding domain of CBP (amino acids 591-670)1, or BSA on association of RNA Pol II activity with phospho(Ser-133) KID resin. Recruitment of Pol II was examined by in vitro transcription assay using MLP template in reactions containing all purified basal factors minus RNA Pol II . $(\alpha \mathrm{CBP}$ and $\alpha$ pol II) RNA Pol II activity contained within immunoprecipitates of CBP and RNA Pol II, respectively. Anti-CTD antiserum was used to immunoprecipitate RNA Pol II activity. MLP transcript and recovery control (CON) as indicated. (B) Western blot analysis of CBP (CBP, top) and RNA Pol II (pol II, bottom) retrieved from GST-phospho(Ser-133) KID resin following affinity selection assay with $0.6 \mathrm{M}$ Bio-Rex fraction (see Fig. 1A). (ON) Twenty percent of total CBP and RNA Pol II activity in $0.6 \mathrm{M}$ Bio-Rex fraction prior to affinity selection assay. Addition of BSA, mutant E1A (mut Ela), KIX, or wild-type E1A (wt Ela) polypeptides to $0.6 \mathrm{M}$ Bio-Rex fraction during affinity selection assay as indicated at top. Anti-CTD antiserum was used to detect RNA Pol II large subunit in Western blot assays. Solid wedges indicate increasing amounts of protein $(2$ $\mu \mathrm{g}$ or $20 \mu \mathrm{g}$ ). Relative mass (in $\mathrm{kD}$ ) indicated at left.

with the $0.6 \mathrm{M}$ Bio-Rex fraction (Fig. 6B). Although the KIX domain could block recruitment of CBP to phospho(Ser-133) KID, neither wild-type nor mutant E1A protein was found to interfere with the phospho(Ser-133) KID-CBP interaction (Fig. 6B). Remarkably, addition of wild-type but not mutant ElA appeared to block the recruitment of RNA Pol II protein to phospho(Ser-133) KID (Fig. 6B), suggesting that E1A represses phospho(Ser-133)
CREB activity by promoting dissociation of CBP from RNA Pol II.

To evaluate whether E1A blocks the association of CBP with RPCs via a direct mechanism, we performed affinity selection assays with a series of GST-CBP polypeptides spanning the CBP protein (Fig. 7A). Following incubation with the $0.6 \mathrm{M}$ Bio-Rex fraction, individual CBP resins were tested for associated RNA Pol II activity by in vitro transcription assay (Fig. 7A) and by Western blot analysis (Fig. 7B). Only CBP polypeptides containing the E1A-binding domain of CBP (amino acids 1805-1890) were found to recruit functional RNA Pol II activity, suggesting that E1A may repress phospho(Ser-133) CREB activity by directly blocking the ability of RNA Pol II to associate with CBP. In this regard, addition of E1A blocked binding of pol II to GST-CBP resins in vitro (not shown).

Should ElA inhibit formation of CBP-RPCs via a direct mechanism, we reasoned that $\mathrm{E} 1 \mathrm{~A}-\mathrm{CBP}$ complexes isolated from the E1A-expressing cell line $293 \mathrm{~T}$ should not contain detectable levels of RNA Pol II. In keeping with the ability of $293 \mathrm{~T}$ cells to activate a cAMP-responsive reporter gene via $\mathrm{CBP}$ in transient transfection assays (T. Nakajima and M. Montminy, unpubl.), albeit at a reduced level, immunoprecipitates of CBP prepared from $293 \mathrm{~T}$ cells were found to contain RNA Pol II by Western blot assay (Fig. 7C, top, lane 3). In contrast, no RNA Pol II was detected in immunoprecipitates of E1A (Fig. 7C, top, lane 4), even though greater amounts of CBP were coprecipitated with the ElA compared with CBP antiserum (Fig. 7C, bottom, cf. lanes 3 and 4). These results support the notion that association of E1A with CBP precludes formation of CBP-RPCs.

\section{Discussion}

Association of E1A with P300 has been shown to block cellular differentiation and to promote cell cycle entry (for review, see Bayley and Mymryk 1994). The ability of E1A to inhibit transcriptional activation via a variety of signal-dependent factors (Arany et al. 1995; Lunblad et al. 1995; Bhattacharya et al. 1996) has supported the notion that E1A may block the ability of P300 and CBP to interact with the transcriptional apparatus.

In this paper we used a partially purified RNA Pol II holoenzyme fraction to reconstitute phospho(Ser-133) CREB activity in vitro. Purified core Pol II does not appear to contain CBP by Western blot assay (M. Montminy, unpubl.), and, correspondingly, core Pol II is unable to support transcriptional induction by phospho(Ser-133) CREB (Fig. 1D). Thus, the association we observed between CBP and Pol II is unlikely to be direct but, rather, via holoenzyme-specific components.

The holo-Pol II complex was found to associate specifically with the kinase-inducible domain following PKA-mediated phosphorylation of CREB at Ser-133 (Fig. 8). Recruitment of this holoenzyme was not sufficient for transcriptional induction of a cAMP-responsive gene, however. Association of CREB with TFIID via the glutamine-rich constitutive activation domain Q2 was also 
C

POL II

A
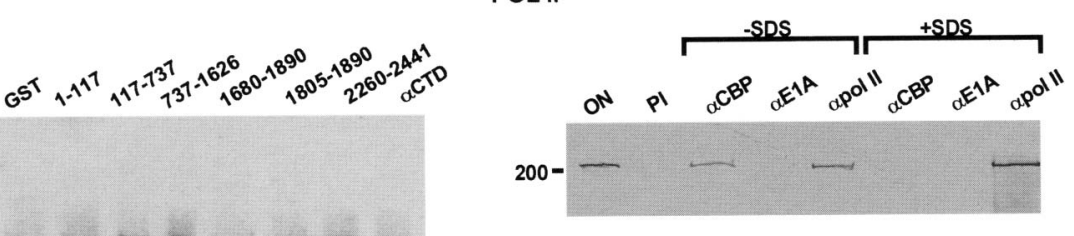

CBP

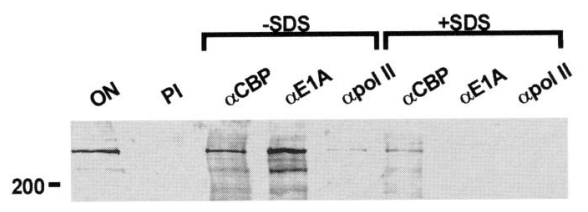

Figure 7. Adenovirus E1A blocks association of CBP with RPCs via a competitive mechanism. (A) CBP associates with RNA Pol II activity via its E1A-binding domain (amino acids 1805-1890). In vitro transcription assay of RNA Pol II activity recovered from $0.6 \mathrm{M}$ Bio-Rex fraction following affinity selection with a series of GST-CBP polypeptide resins. Following the pull-down reaction, resins were washed and analyzed for RNA Pol II activity by in vitro transcription assay with all purified basal factors except RNA Pol II. Amino acid endpoints for each CBP polypeptide are indicated at top. (GST) Control assay with GST alone; $(\alpha$ CTD) RNA Pol II activity recovered from immunoprecipitates of RNA Pol II prepared with antiserum against the carboxy-terminal domain of RNA Pol II large subunit. Recovery control (CON) indicated. $(B)$ Western blot analysis of RNA Pol II large subunit protein (POL II) recovered from $0.6 \mathrm{M}$ Bio-Rex fraction following affinity selection assay with a series of GST-CBP polypeptide resins. Inclusive amino acid endpoints for each polypeptide are indicated at top. (ON) Onput. $(C)$ Western blot analysis of RNA Pol II (POL II) and CBP in adenovirus E1A-expressing $293 \mathrm{~T}$ cells. Immunoprecipitations were performed in the absence or presence of $1 \%$ SDS as indicated. ( $\alpha$ CTD) Anti-RNA Pol II antiserum; (M73) monoclonal anti-E1A antiserum; (5614) polyclonal anti-CBP antiserum developed against the CREB-binding domain of CBP; (PI) preimmune serum; (ON) $20 \%$ of total protein from $293 \mathrm{~T}$ cell extracts used in immunoprecipitation reactions.

required. Thus, two signals were critical for target gene expression in response to cAMP stimulation (Fig. 8). The importance of both signals for transcriptional activation is further illustrated by the CREB-related CREM- $\alpha$,

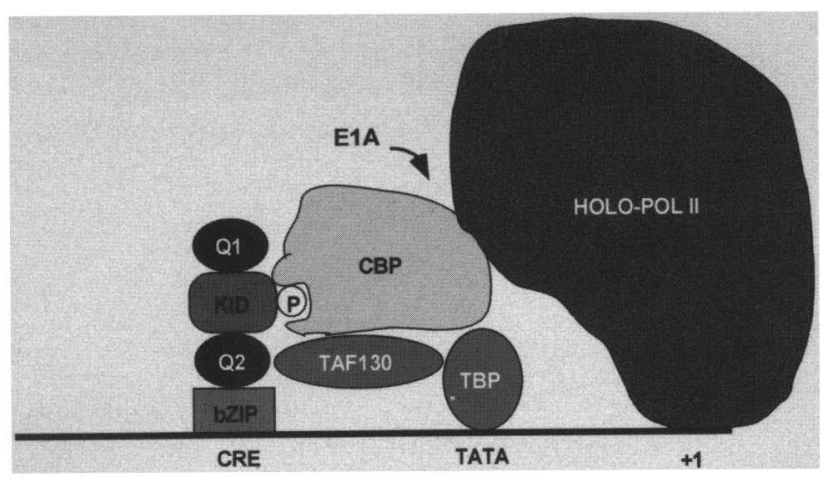

Figure 8. Model for activation of cAMP-responsive genes by CREB. Phospho(Ser-133) CREB stimulates transcription of cAMP-responsive genes by associating with TFIID and RNA Pol II holoenzyme complexes. Following hormonal induction, cAMP stimulates phosphorylation of CREB at Ser-133. (Q1, KID, Q2, and bZIP) Domains of CREB. Ser-133 phosphorylation enhances CREB activity by recruiting RNA Pol II holoenzyme complexes via CBP. A glutamine-rich region in CREB, termed Q2, stimulates recruitment of TFIID via its interaction with $\mathrm{hTAF}_{\mathrm{II}} 130$. The adenovirus E1A oncoprotein is proposed to inhibit activation of cellular genes by disrupting CBP-RNA Pol II holoenzyme complexes.
CREM- $\beta$, and CREM- $\epsilon$ proteins that contain a KID region yet appear to function as transcriptional repressors owing to the absence of a Q2 activation domain (Foulkes and Sassone-Corsi 1992; Brindle et al. 1993).

In addition to CREB, a growing number of signal-dependent factors appear to require CBP and P300 for target gene activation. Our results indicate that, by analogy with phospho(Ser-133) CREB, the recruitment of CBP alone does not suffice for transcriptional activation. We speculate that these signal-dependent factors will contain bipartite activation domains that, in addition to associating with CBP, are also capable of recruiting TFIID.

In addition to $\mathrm{E} 1 \mathrm{~A}$, a number of regulators such as the SV40 large $T$ antigen and the mitogen-activated $\mathrm{pp} 90_{\mathrm{RSK}}$ have been shown to repress cellular gene expression by interacting with the E1A-binding domain of P300 and CBP (Eckner et al. 1996; Nakajima et al. 1996). Our data indicate that these regulators may similarly inhibit transcription of signal-dependent genes by regulating the composition of RPCs.

The same surface of CBP that binds to Pol II also appears to interact with a histone acetylase, referred to as pCAF (Yang et al. 1996). pCAF has been proposed to mediate the transcriptional effects of $\mathrm{CBP}$ via a nucleosome displacement model in which signal-dependent factors like CREB may gain access to their target sites on chromatinized templates. pCAF did not appear to fractionate with the CBP-Pol II complex during Bio-Rex 70 column chromatography (T. Nakajima, J. Parvin, and M. Montminy, unpubl.), however, suggesting that the CBP-pCAF 
complex is distinct from the CBP-Pol II complex. It will be of interest to determine whether CBP mediates different cellular activities depending on its association with the RNA Pol II holoenzyme or with pCAF.

\section{Materials and methods \\ Expression and purification of general transcription factors and recombinant polypeptides}

TFIIB, TBP, TFIIE, and TFIIF cDNAs were expressed in Escherichia coli, and the recombinant proteins were purified as described previously (Parvin et al. 1994). Recombinant TFIIA-expressing constructs were a generous gift of R. Roeder, and TFIIA was prepared as per the published protocol (DeJong and Roeder 1993; DeJong et al. 1995). TFIID was obtained from Hela wholecell extracts by collecting an $0.5-1.0 \mathrm{M}$ protein peak from a phosphocellulose column. Highly purified epitope-tagged TFIID was obtained from a stably transfected line of Hela cells expressing influenza HA epitope-tagged TBP as described previously (Zhou et al. 1992). TFIIH was prepared from HeLa whole-cell extracts as described previously (Parvin et al. 1994). Core RNA Pol II was purified from calf thymus as indicated previously (Thompson et al. 1990). In assays for basal transcription, TFIID was replaced by recombinant yeast TBP subunit. Lack of crosscontamination between individual general transcription factors was verified by in vitro transcription assay with an adenovirus MLP template (not shown).

Experiments with E1A were performed with recombinant polypeptides spanning exon 1 (amino acids 1-139) and containing CR1 of the Ad5 E1A gene, a region that is sufficient for binding to and inhibiting CBP/ P300 activity (Egan et al. 1988). The E1A mutant gene dl 1101 contains a deletion from amino acid 4 to 25 that disrupts binding of E1A to CBP and P300 (Egan et al. 1988). Wild-type and mutant E1A polypeptides were expressed as GST fusion proteins in BL21(DE3) E. coli, purified on glutathione-Sepharose beads, and then cleaved from GST with thrombin. For transcription assays, $2 \mu \mathrm{g}$ or $20 \mu \mathrm{g}$ of mutant or wild-type ElA polypeptide was added to each reaction. The CREB-binding domain of CBP (KIX, amino acids 591-670 of CBP) was expressed as a GST fusion protein in pGEX-KT and purified as described previously. KIX polypeptide was cleaved with thrombin and concentrated on a centricon filter. For transcription assays, $2 \mu \mathrm{g}$ or $20 \mu \mathrm{g}$ of KIX polypeptide was added to each reaction.

\section{In vitro basal transcription assays with MLP template}

In vitro transcription assays were performed in a reaction volume of $25 \mu$ l containing $100 \mathrm{~mm}$ potassium acetate, $6 \mathrm{~mm}$ magnesium acetate, $20 \mathrm{~mm}$ Tris-acetate $(\mathrm{pH} 7.9), 1 \mathrm{~mm}$ EDTA, $20 \%$ glycerol, $100 \mu \mathrm{M}$ each ATP and UTP, $50 \mu \mathrm{M}$ 3'-O methyl GTP, $3 \mu \mathrm{M} \mathrm{CTP}, 1 \mathrm{~mm}$ DTT, $2.5 \mu \mathrm{g} / \mathrm{ml}$ of plasmid DNA template, and $10 \mu \mathrm{Ci}$ of $\left[\alpha-{ }_{-}^{32} \mathrm{P}\right] \mathrm{CTP}(800 \mathrm{Ci} / \mathrm{mmole}$, DuPont NEN) plus transcription factors. For each reaction, $30 \mathrm{ng}$ TFIIB, $8 \mathrm{ng}$ of yTBP, 4 $\mathrm{ng}$ of TFIIE ( $3 \mathrm{ng}$ of p56 and $1 \mathrm{ng}$ of p34), $100 \mathrm{ng}$ of TFIIF (50 ng each of RAP 30 and RAP 74), 100 ng of core RNA Pol II, and 0.25 $\mu l$ of the TFIIH fraction was used. Reaction components were mixed on ice and allowed to incubate for $60 \mathrm{~min}$ at $30^{\circ} \mathrm{C}$.

For transcription reactions containing immune complexes, avidin, or glutathione beads, pull-down assays were performed overnight at $4^{\circ} \mathrm{C}$ on a rotator in $200 \mu$ l of final volume using 20 $\mu \mathrm{g}$ of $0.6 \mathrm{M}$ Bio-Rex 70 or $1 \mu \mathrm{l}$ of TFIID fraction with $2 \mu \mathrm{g}$ (15 $\mu \mathrm{l}$ of beads) of biotinylated CREB or GST fusion proteins plus binding buffer that contained $100 \mathrm{~mm}$ potassium acetate, $10 \mathrm{~mm}$
Tris-acetate $(\mathrm{pH} 7.8), 2 \mathrm{~mm}$ magnesium acetate, $0.1 \%$ NP-40, 1 mM sodium fluoride, $1 \mathrm{~mm}$ DTT, and $10 \mu \mathrm{M}$ PMSF. Pull-down assays for RNA Pol II were performed with $0.1 \mu \mathrm{g}\{1 \mu 1\}$ of carboxy-terminal domain antibody (Promega). Prior to transcription reaction, immune complexes, avidin, and glutathione beads were washed four times with $300-500 \mu$ l of binding buffer. Transcription reactions were performed on the moist beads.

Reactions were terminated by adding $200 \mu 1$ of stop mix containing $0.5 \%$ SDS, $7 \mathrm{~m}$ urea, $2.5 \mathrm{~mm}$ EDTA, $100 \mathrm{~mm}$ lithium chloride, $380 \mathrm{~mm}$ ammonium acetate, and $10 \mu \mathrm{g}$ of tRNA. Samples were extracted with phenol-chloroform and precipitated with ethanol. Following resuspension with loading buffer, ${ }^{32} \mathrm{P}$-labeled transcripts were resolved by electrophoresis over $6 \%$ urea-polyacrylamide gels.

\section{Transcriptional activation assays with CRE template}

The adenovirus MLP used for activation assays with phospho(Ser-133) CREB and CREB ( $3 \times$ CRE-MLP) contained three CRE sites extending from -56 to -32 of the somatostatin gene inserted upstream of the MLP TATA box. Biotinylated recombinant CREB protein was expressed from a Pin Point Xa bacterial expression vector (Promega) and was phosphorylated with purified PK-A catalytic subunit (gift of Susan Taylor, University of California, San Diego). Following kinase reaction, phospho(Ser-133) CREB beads were washed with $100 \mathrm{~mm} \mathrm{KCl,} 10$ $\mathrm{mM}$ Tris ( $\mathrm{pH} 7.8$ ) to remove unincorporated ATP and PK-A. For activation assays with $3 \times$ CRE-MLP template $(2.5 \mu \mathrm{g} / \mathrm{ml}), 60 \mathrm{ng}$ of CREB and phospho(Ser-133) CREB was added to transcription reactions for a final concentration of $60 \mathrm{nM}$. In activation assays, RNA Pol II activity was supplied by addition of $2 \mu \mathrm{g}(1 \mu \mathrm{l})$ of the Bio-Rex $700.6 \mathrm{M}$ potassium acetate column fraction containing the CBP-RNA Pol II holoenzyme complex. TFIID activity was supplied by addition of crude Hela TFIID fraction $(1-2 \mu 1)$. Recombinant TFIIA was used only for activation assays $(1 \mu l)$ and was prepared as described (DeJong and Roeder 1993; DeJong et al. 1995). TFIIB, TFIIE, TFIIF, and TFIIH proteins were added as described above for basal transcription assays with MLP template. CRE-MLP template was preincubated with CREB, $0.6 \mathrm{M}$ Bio-Rex 70 fraction, and TFIID complex for $20 \mathrm{~min}$ on ice prior to the transcription reaction. For immunoneutralization assay with hTAF130 antiserum, preimmune serum or hTAF 130 antibody $(0.5 \mu \mathrm{g})$ (gift of $\mathrm{N}$. Tanese, NYU Medical Center, New York, NY) was added to crude Hela TFIID fraction $(2 \mu \mathrm{l})$ on ice for $30 \mathrm{~min}$ prior to addition of CREB and $0.6 \mathrm{M}$ Bio-Rex 70 fraction.

\section{Sucrose gradient analysis}

For sucrose gradient purification of the RNA Pol II holoenzyme complex, $6 \mathrm{ml}(12 \mathrm{mg})$ of the $0.6 \mathrm{M}$ Bio-Rex 70 column fraction was loaded onto $28 \mathrm{ml}$ of a $10 \%-60 \%$ sucrose gradient containing $0.2 \mathrm{M}$ potassium acetate, $20 \mathrm{~mm}$ Tris-acetate $(\mathrm{pH} 7.9), 1 \mathrm{~mm}$ EDTA, and $0.1 \%$ NP-40. Samples were centrifuged in a swinging bucket SW28 rotor at $25,000 \mathrm{rpm}$ for $16 \mathrm{hr}\left(4^{\circ} \mathrm{C}\right)$. Sedimentation fractions $(1 \mathrm{ml})$ were then collected and were used for Western blot and in vitro transcription assays. For activation assays with $3 \times$ CRE-MLP template (Fig. $2 B$ ), $0.5 \mu$ l of fraction 15 , containing $0.4 \mu \mathrm{g}$ of RNA Pol II holoenzyme purified 70 - to 100 -fold relative to total protein, was added to each transcription reaction. In these assays, the highly purified epitope-tagged TFIID was used. In vitro transcription assays of the sucrose gradient fractions for TFIIH activity were performed with linearized MLP template plus $3 \mu 1$ of each sucrose gradient fraction. 


\section{Acknowledgments}

We thank N. Tanese and D. Saluja for the $\mathrm{hTAF}_{\mathrm{II}} 130 \mathrm{cDNA}$ and antiserum and for sharing results on $\mathrm{hTAF}_{\mathrm{II}} 130-\mathrm{CREB}$ interactions prior to publication. We thank D. Chao and R.A. Young for the gift of anti-SRB7 antiserum and E. Nigg for anti-CDK8 antiserum. This work was supported by National Institutes of Health (NIH) grants GM37828 and CA54418 (T.N., C.U., M.M.). J.D.P. is supported by NIH grant GM53504, by the American Cancer Society, Massachusetts Division, Inc., and by a Junior Faculty Research Award from the American Cancer Society. S.A. is supported by an institutional NIH postdoctoral training grant. T.N. is also supported by the Uehara Memorial Foundation and by the Otsuka Pharmaceutical Company, and C.U. is supported by the Japanese Ministry of Education, Science, and Culture.

The publication costs of this article were defrayed in part by payment of page charges. This article must therefore be hereby marked "advertisement" in accordance with 18 USC section 1734 solely to indicate this fact.

\section{References}

Arany, Z., D. Newsome, E. Oldread, D. Livingston, and R. Eckner. 1995. A family of transcriptional adaptor proteins targeted by the E1A oncoprotein. Nature 374: 8184.

Arias, J., A. Alberts, P. Brindle, F. Claret, T. Smeal, M. Karin, J. Feramisco, and M. Montminy. 1994. Activation of cAMP and mitogen responsive genes relies on a common nuclear factor. Nature 370: 226-228.

Bayley, S. and J. Mymryk. 1994. Adenovirus E1A proteins and transformation. Int. I. Oncol. 5: 425-444.

Bhattacharya, S., R. Eckner, S. Grossman, E. Oldread, Z. Arany, A. D'Andrea, and D. Livingston. 1996. Cooperation of STAT2 and P300/CBP in signaling induced by interferon-a. Nature 383: 343-347.

Brindle, P., S. Linke, and M. Montminy. 1993. Analysis of a PK-A dependent activator in CREB reveals a new role for the CREM family of repressors. Nature 364: 821-824.

Chakravarti, D., V. LaMorte, M. Nelson, T. Nakajima, I. Schulman, H. Juguilon, M. Montminy, and R. Evans. 1996. Role of $\mathrm{CBP} / \mathrm{P} 300$ in nuclear receptor signaling. Nature 383: 99-103.

Chrivia, J.C., R.P. Kwok, N. Lamb, M. Hagiwara, M.R. Montminy, and R.H. Goodman. 1993. Phosphorylated CREB binds specifically to the nuclear protein CBP. Nature 365: $855-859$.

DeJong, J. and R. Roeder. 1993. A single cDNA, hTFIIA/a, encodes both the p35 and p19 subunits of TFIIA. Genes \& Dev. 7: $2220-2234$.

DeJong, J., R. Bernstein, and R. Roeder. 1995. Human general transcription factor TFIIA: Characterization of a cDNA encoding the small subunit and requirement for basal and activated transcription. Proc. Natl. Acad. Sci. 92: 3313-3317.

Eckner, R., M.E. Ewen, D. Newsome, M. Gerdes, J.A. DeCaprio, J.B. Lawrence, and D.M. Livingston. 1994. Molecular cloning and functional analysis of the adenovirus E1A-associated $300-\mathrm{kD}$ protein $(\mathrm{p} 300$ ) reveals a protein with properties of a transcriptional adaptor. Genes \& Dev. 8: 869-884.

Eckner, R., J. Ludlow, N. Lill, E. Oldread, Z. Arany, N. Moditahedi, J. DeCaprio, D. Livingston, and J. Morgan. 1996. Association of $\mathrm{P} 300$ and CBP with simian virus 40 large $\mathrm{T}$ antigen. Mol. Cell. Biol. 16: 3454-3464.

Egan, C., T. Jelsma, J. Howe, S. Bayley, B. Ferguson, and P. Branton. 1988. Mapping of cellular protein-binding sites on the products of early-region $1 \mathrm{~A}$ of human adenovirus type 5 .
Mol. Cell. Biol. 8: 3955-3959.

Ferreri, K., G. Gill, and M. Montminy. 1994. The cAMP regulated transcription factor CREB interacts with a component of the TFIID complex. Proc. Nat1. Acad. Sci. 91: 1210-1213.

Foulkes, N.S. and P. Sassone-Corsi. 1992. More is better: Activators and repressors from the same gene. Cell 68: 411-414.

Gonzalez, G.A. and M.R. Montminy. 1989. Cyclic AMP stimulates somatostatin gene transcription by phosphorylation of CREB at Serine 133. Cell 59: 675-680.

Gonzalez, G.A., P. Menzel, J. Leonard, W.H. Fischer, and M.R. Montminy. 1991. Characterization of motifs which are critical for activity of the cyclic AMP-responsive transcription Factor CREB. Mol. Cell. Biol. 11: 1306-1312.

Hagiwara, M., A. Alberts, P. Brindle, J. Meinkoth, J. Feramisco, T. Deng, M. Karin, S. Shenolikar, and M. Montminy. 1992. Transcriptional attenuation following cAMP induction requires PP-1-mediated dephosphorylation of CREB. Cell 70: 105-113.

Janknecht, R. and A. Nordheim. 1996. Regulation of the c-fos promoter by the ternary complex factor Sap-1a and its coactivator CBP. Oncogene 12: 1961-1969.

Kamei, Y., L. Xu, T. Heinzel, J. Torchia, R. Purokawa, B. Gloss, S. Lin, R. Heyman, D. Rose, C. Glass, and M.G. Rosenfeld. 1996. A CBP integrator complex mediates transcriptional activation and AP-1 inhibition by nuclear receptors. Cell 85: 403-414.

Kee, B., J. Arias, and M. Montminy. 1996. Adaptor mediated recruitment of RNA polymerase II to a signal dependent activator. J. Biol. Chem. 271: 2373-2375.

Kwok, R., J. Lundblad, J. Chrivia, J. Richards, H. Bachinger, R. Brennan, S. Roberts, M. Green, and R. Goodman. 1994. Nuclear protein CBP is a coactivator for the transcription factor CREB. Nature 370: 223-226.

Lunblad, J., R. Kwok, M. Laurance, M. Harter, and R. Goodman. 1995. Adenoviral E1A associated protein p300 as a functional homolog of the transcriptional co-activator CBP. $\mathrm{Na}$ ture 374: 85-88.

Maldonado, E., R. Shiekhattar, M. Sheldon, H. Cho, R. Drapkin, P. Rickert, E. Lees, C. Anderson, S. Linn, and D. Reinberg. 1996. A human RPC associated with SRB and DNA-repair proteins. Nature 381: 86-89.

Nakajima, T., A. Fukamizu, J. Takahashi, F. Gage, T. Fisher, J. Blenis, and M. Montminy. 1996. The signal dependent coactivator CBP is a nuclear target for pp90RSK. Cell 86: $465-$ 474.

Parker, D., K. Ferreri, T. Nakajima, V. LaMorte, R. Evans, S. Koerber, C. Hoeger, and M. Montminy. 1996. Phosphorylation of CREB at Ser133 induces complex formation with CBP via a direct mechanism. Mol. Cell. Biol. 16: 694-703.

Parvin, J., B. Shykind, R. Meyers, J. Kim, and P. Sharp. 1994. Multiple sets of basal factors initiate transcription by RNA polymerase II. J. Biol. Chem. 269: 18414-18421.

Tanese, N., D. Saluja, M. Vassallo, J. Chen, and A. Admon. 1996. Molecular cloning and analysis of two subunits of the human TFIID complex: hTAFII130 and hTAFII100. Proc. Natl. Acad. Sci. 93: 13611-13616.

Thompson, N., D. Aronson, and R. Burgess. 1990. Purification of eukaryotic RNA polymerase II by immunoaffinity chromatography. I. Biol. Chem. 265: 7069-7077.

Yang, X., V. Ogryzko, J. Nishikawa, B. Howard, and Y. Nakatani. 1996. A P300/CBP associated factor that competes with the adenoviral oncoprotein E1A. Nature 382: 319-322.

Zhou, Q., P.M. Lieberman, T. Boyer, and A. Berk. 1992. HoloTFIID supports transcriptional stimulation by diverse activators and from a TATA-less promoter. Genes \& Dev. 6: 1964-1974. 


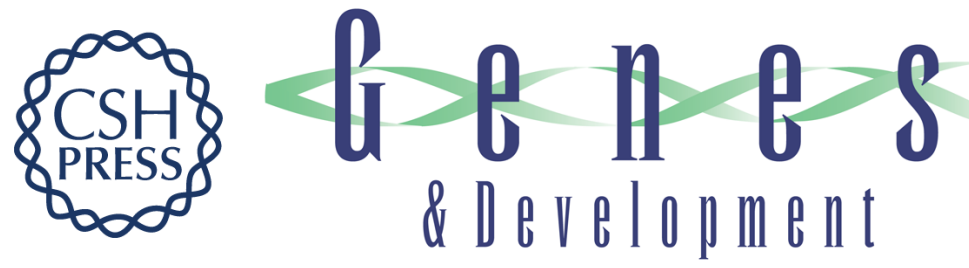

\section{Analysis of a cAMP-responsive activator reveals a two-component mechanism for transcriptional induction via signal-dependent factors.}

T Nakajima, C Uchida, S F Anderson, et al.

Genes Dev. 1997, 11:

Access the most recent version at doi:10.1101/gad.11.6.738

References This article cites 30 articles, 13 of which can be accessed free at: http://genesdev.cshlp.org/content/11/6/738.full.html\#ref-list-1

License

Email Alerting Service

Receive free email alerts when new articles cite this article - sign up in the box at the top right corner of the article or click here.

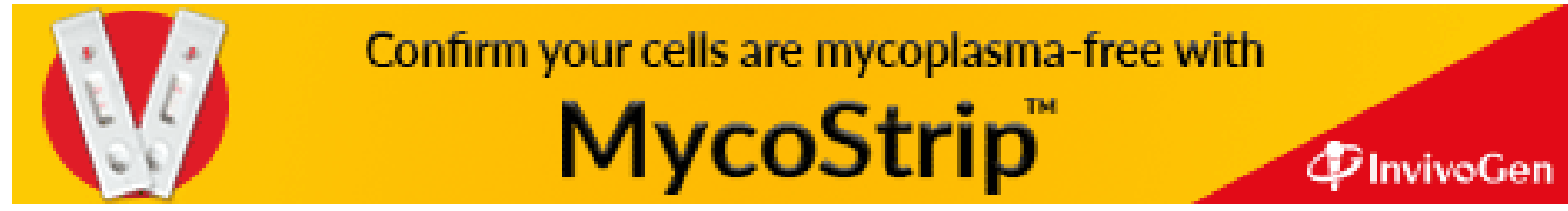

